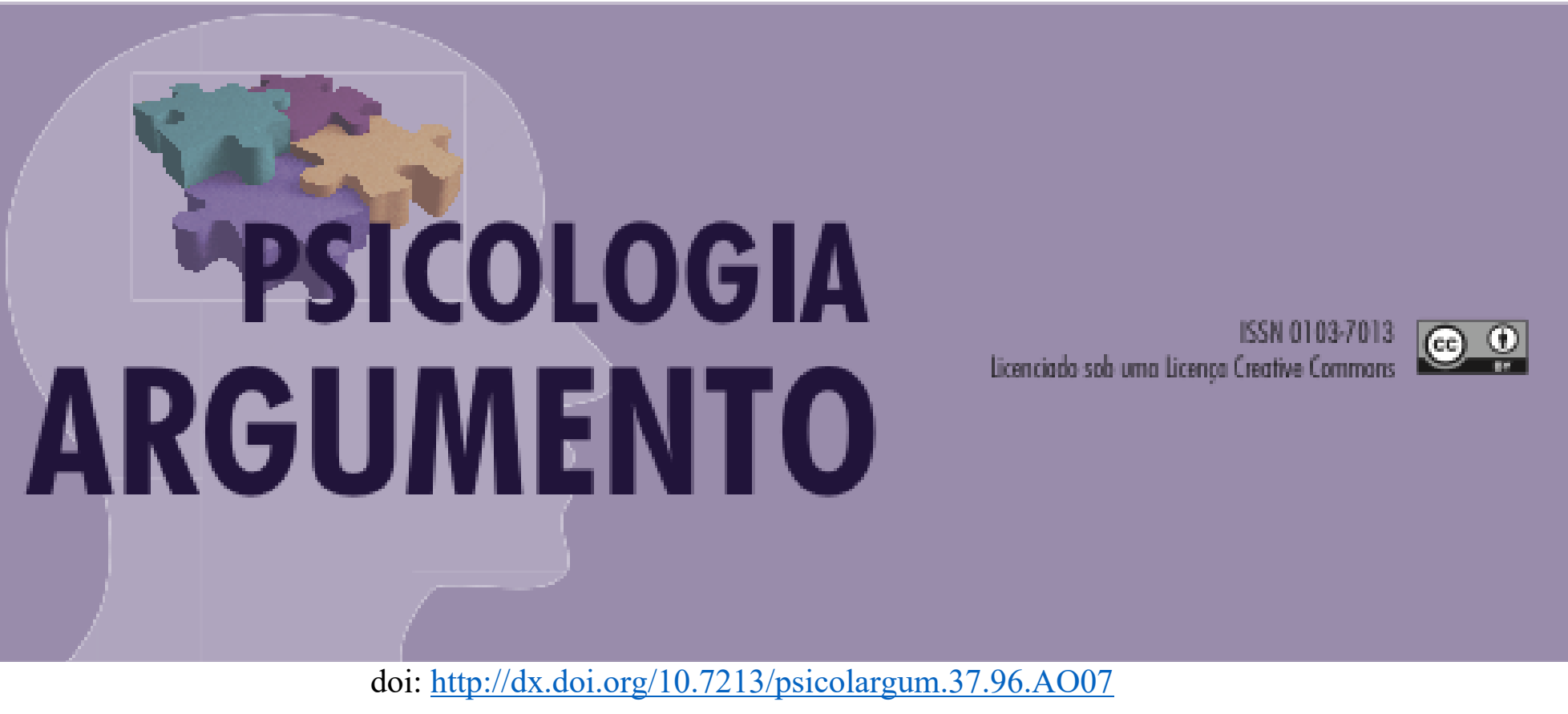

\title{
Clima escolar autoritativo: adaptação e evidências de validade entre estudantes brasileiros
}

Authoritative school climate: adaptation and validity evidence among brazilian students

Clima escolar autorizado:adaptación y evidencia de validez entre estudiantes brasileños

\author{
Josafá Moreira da Cunha \\ https://orcid.org/0000-0002-4003-6847
}

Docente Departamento de Teoria e Fundamentos da Educação, Universidade Federal do Paraná.

Email: josafas@gmail.com

Hellen Tsuruda do Amaral https://orcid.org/0000-0003-4511-4426

Universidade Federal do Paraná. Email: hellentsuruda@gmail.com

Ana Moreira Borges de Macedo

https://orcid.org/0000-0002-1213-4191

Universidade Federal do Paraná. Email: anamacedo266@hotmail.com

\section{Resumo}

O clima escolar é um fator fundamental na qualidade da vida escolar, sendo relevante para o bem estar dos estudantes. Nesse contexto duas dimensões destacam-se como sendo chave para mensuração do clima: estrutura disciplinar e suporte. Representam respectivamente a aplicação justa e estrita das regras da escola e a percepção dos estudantes quanto ao apoio de seus professores. A ampliação sobre seu estudo pode favorecer o desenvolvimento das escolas e dos 
estudantes ao contribuir na criação de um ambiente apropriado para esse fim. O presente estudo, portanto, teve como objetivos a adaptação para contexto brasileiro e a obtenção de evidências de confiabilidade e validade dos construtos de estrutura disciplinar e suporte da Escala de Avaliação do Clima Escolar Autoritativo (EACEA), versão para estudantes. Os dados foram coletados com 708 estudantes do segundo ciclo do ensino fundamental e do ensino médio de sete escolas públicas da cidade de Curitiba, Paraná. Dentre os participantes, 55,1\% se autodeclaram brancos e $53,0 \%$ do sexo feminino. As idades variaram entre 9 e 21 anos (média=13,4; d.p.=1,9). Os procedimentos envolveram as etapas de tradução, avaliação qualitativa da escala, Retrotradução, coleta de dados e análise fatorial confirmatória. Também foi executada análise de validade relacionada ao critério. Os principais resultados demonstraram que a escala obteve índices próximos aos valores com a amostra geral e, para a divisão entre grupos acadêmicos específicos, valores aceitáveis no construto de suporte. Na amostra geral a estrutura disciplinar foi um preditor negativo e significativo para a vitimização. Os resultados indicam que a versão brasileira da EACEA pode ser um instrumento adequado para avaliar o clima escolar.

Palavras-chave: clima escolar autoritário; validação de escalas; vitimização por pares; assédio moral.

\begin{abstract}
School climate is a determining factor in the quality of school life and is fundamental to the wellbeing of students. In this context, two dimensions stand out as key to climate measurement: disciplinary structure and support. They represent, respectively, the fair and strict application of school rules and the students' perception of the support of their teachers. Their investigation can favor the development of schools and students, by contributing to the creation of an appropriate environment. Therefore, the present study aimed to adapt for the Brazilian context and obtaining evidence of reliability and validity of the constructs of disciplinary structure and support of the Authoritative School Climate Rating Scale (ASCRS), version for students. Data were collected with 708 students from the second cycle of elementary and high school education in seven public schools in the city of Curitiba, Paraná. Among the participants, $55.1 \%$ were self-described whites and $53.0 \%$ were female. The ages ranged from 9 to 21 years (mean=13.4, s.d. $=1.9$ ). The procedures involved the steps of translation, qualitative scale evaluation, Retrotranslation, data collection and confirmatory factor analysis. Criterion-related validity analysis was also performed. The main results showed that the scale obtained values close to the general sample and acceptable values in the support construct for the division between specific academic groups. In the general sample the disciplinary structure was a negative and significant predictor for victimization. The results indicate that the Brazilian version of the EACEA can be an adequate instrument to evaluate the school climate.
\end{abstract}

Keywords: Authoritative School Climate; Validation of Scales; Peer Victimization; Bullying.

\title{
Resumen
}

O clima escolar é um fator fundamental na qualidade da vida escolar, sendo relevante para o bem estar dos estudantes. Nesse contexto duas dimensões destacam-se como sendo chave for mensuração do clima: estrutura disciplinar e suporte. Representam respectivamente a aplicação justa y estrita das regras da escola e a percepção dos estudantes quanto ao apoio de seus professores. Una ampliação sobre seu estudo pode favorecer o desenvolvimento das escolas y dos estudantes ao contribuir a la criação de um ambiente apropiado para esse fim O presente estudo, portanto, teve como objetivos a adaptção for context brasileiro and a obtenção of evidências of confiabilidade and validade dos construtos of estrutura disciplinar and suporte da Escala de Avaliação do Clima Escolar Autoritativo (EACEA), versão para estudantes. Os dados foram coletados com 708 estudantes do segundo ciclo do ensino fundamental e do ensino médio de sete escolas públicas da cidade de Curitiba, Paraná. Dentre os participantes, 55,1\% se autodeclaram brancos e 53,0\% do sexo feminino. As idades variaram entre 9 y 21 años (medio $=13,4 ;$ d.p. = 1,9). Los procedimientos se envuelven como etapas de traducción, avaliação qualitativa da escala, Retrotradução, coleta de dados e análise fatorial confirmamatória. También se ejecuta análise de validación relacionada con la crítica. Los principales resultados muestran una escala obteve índices próximos aos valores com amostra general, para una división 
entre grupos académicos especificos, valores aceitáveis no construto de suporte. Na amostra geral a estrutura disciplinar foi um preditor negativo e significativo para a vitimização. Os resultados indicam que a versão brasileira da EACEA pode ser um instrumento adequado for avaliar or clima escolar.

Palavras-chave: clima escolar autoritário; validação de escalas; vitimização por pares; Assédio moral.

\section{Introdução}

O estudo sobre os efeitos do ambiente social escolar no desenvolvimento e adaptação de estudantes por meio da abordagem do clima escolar tem sido foco crescente (Thapa et al., 2013; Vinha et al., 2016), podendo contribuir para a compreensão das desigualdades no desempenho acadêmico observadas entre estudantes de diferentes escolas Brasileiras (Franco et al., 2007; Alves, Franco, 2008; Oliveira et al, 2013). O clima é determinante na qualidade da vida escolar e associa-se ao bem estar geral dos estudantes, tratando-se, portanto, de um conceito chave para o planejamento escolar e implementação de estratégias que busquem a melhoria do ambiente escolar (Vinha et al, 2016).

Embora ainda não exista consenso amplo na literatura a respeito da definição do clima escolar (Thapa et al, 2013), duas dimensões têm sido destacadas como categorias fundamentais na análise sobre a influência do ambiente escolar: a estrutura disciplinar e o suporte do ambiente escolar. Primeiro, a estrutura considerada por alguns estudos como a aplicação rigorosa e justa das regras escolares (Gregory et al., 2010; Konold et al., 2014). Além desta, o suporte, bastante mencionado como sendo a percepção dos estudantes sobre quanto seus professores importam-se com eles e demonstram respeito e disposição para ajudar. A combinação de tais fatores está associada a melhores indicadores de interação social no ambiente escolar, como a redução da vitimização de estudantes (Cornell, Shukla, Konold, 2015) e também de professores (Gregory, Cornell, Fan, 2012), com impactos positivos incluindo a redução de comportamentos de risco (Cornell \& Huang, 2016) e maior engajamento e desempenho acadêmico entre estudantes (Cornell, Shukla \& Konold, 2016).

A ampliação e divulgação do estudo sobre a mensuração do clima a partir desses dois fatores poderá tornar o seu acesso comum às escolas e assim fornecer maiores subsídios aos psicólogos e demais profissionais desse ambiente. Estes, a partir dessas informações poderão criar e implementar programas para facilitar o desenvolvimento 
contínuo da escola, favorecendo um ambiente de inclusão e tolerância que ofereça suporte e segurança aos estudantes e assim contribua mais intensamente para seu desenvolvimento apropriado (Cornell \& Bradshaw, 2015).

Clima escolar autoritativo

O ambiente escolar é caracterizado por diversas formas de interação, sendo percebido e compreendido como uma influência fundamental para a compreensão dos processos de ajustamento escolar e para os comportamentos dos estudantes nesse ambiente (Cohen, 2009; Huang et al., 2015; Cornel \& Huang, 2016). Nas últimas duas décadas, aumentou expressivamente a quantidade de estudos enfatizando a importância do clima escolar (Bear et al 2016). Este é baseado em experiências vividas no ambiente escolar, engloba valores, relações interpessoais, ensino e aprendizagem e mesmo a estrutura da escola (Thapa, Cohen, Guffey \& D`Alessandro, 2013). Grande parte das definições sobre o clima escolar, apesar de diferirem em alguns aspectos, incluem a influência das relações interpessoais e também o sentimento de segurança dentro desse ambiente e relacionam o clima ao envolvimento dos estudantes com a escola e seu ajuste social e emocional (George et al,2016).

O índice de Desenvolvimento da Educação Básica (ideb) e o Pisa, avaliações com o intuito de diagnosticar a qualidade do aprendizado e o desempenho escolar, mensuraram a qualidade do clima escolar, os índices de evasão e desempenho acadêmico, dentre outras características do ambiente escolar. A partir dessa avaliação demonstrou-se a relação direta entre esses três fatores, além da indicação das pesquisas de que um clima positivo esteja relacionado a menor violência escolar (Vinha et al., 2016).

Ou seja, a promoção de um clima escolar positivo está associada à redução das situações de agressão (Gage, Prykanowski \& Larson, 2014; Konold \& Cornell, 2015), em que conflitos podem ser abordados por meio de estratégias não-violentas, no qual estudantes e outros atores da instituição escolar sentem-se protegidos e seguros para aprender e ensinar. Dessa forma, além do encorajamento para identificação e intervenção em situações que possam ser prejudiciais, contribui-se para a utilização de estratégias que não abranjam a punição ou suspensão, visto que estas estão associadas a desistências escolares, desengajamento e mesmo ao fracasso escolar (Nekevasil \& Cornell, 2015).

Nesse contexto a promoção de um clima escolar positivo é apontada como fator que pode diminuir a probabilidade de intimidações, melhorar as relações ao diminuir a vitimização entre pares, melhorar o desempenho acadêmico e desenvolvimento social 
(Gage \& Larson, 2014), auxiliar no relato e resolução de situações de agressão e favorecer as interações positivas (Cornell \& Bradshaw, 2015). Isso, pois o clima escolar autoritativo oferece condições apropriadas para uma boa e segura interação entre os estudantes e sua cooperação com as regras da escola. Em consequência auxilia para evitar situações de vitimização e violência entre os participantes do contexto escolar (Vinha et al., 2016).

Considerando os fatores expostos, é importante retomar que o clima escolar é conceituado de distintos modos pela literatura, prevalecendo à menção à qualidade das relações interpessoais e interações dos estudantes com os outros participantes do contexto escolar e entre eles (Cornell \& Huang, 2016; Cornell, Shukla \& Konold, 2016), além das experiências na vida escolar, segurança, reflexão sobre as regras, objetivos, práticas de ensino (Cohen, 2009; Huang et al., 2015; Cornel \& Huang, 2016). Todavia, apesar dessa prevalência não se encontra até o momento um acordo quanto à sua definição, dimensões ou mesmo quanto à maneira de mensurá-lo (Cornell \& Huang, 2016; Cornell, Shukla \& Konold, 2016). Aponta-se que a dificuldade em sua mensuração mais precisa, em parte, deve-se a inexistência de um consenso na sua conceituação (Cornel et Huang, 2016).

A partir desse cenário, o presente estudo propõe a utilização da teoria do clima escolar autoritativo, já mencionada por Stockard e Mayberry (1992) com a denominação de estrutura do clima e teoria da disciplina autoritativa, referindo-se a visão de dois elementos chaves para a percepção do clima escolar: estrutura e suporte (Bear et al 2016). Tal teoria propôs-se a averiguar o modelo do clima escolar autoritativo como uma possibilidade de aferir e analisar as relações entre os elementos chave do clima escolar. Esse modelo consiste em um quadro teórico que favorece a precisão e consequente mensuração das propriedades de um clima que se possa conceituar como positivo (Cornel \& Huang, 2016). A referida teoria provém do estudo sobre os Estilos Parentais, previamente proposto por Baumrind (1966), que conta com parâmetros para avaliar a relação entre pais e filhos denominados "exigência" e "responsividade". A primeira dimensão representa a maneira como os pais controlam o comportamento dos filhos, a insistência e cobrança dos pais para que a criança atinja as expectativas de um comportamento apropriado, com a imposição ou não de regras e limites para isso. Já a segunda dimensão abrange a sensibilidade dos pais quanto às necessidades da criança, o acolhimento e afeto que os responsáveis propiciam, além do suporte emocional e a autonomia promovida (Baumrind, 1966; Cornel \& Huang, 2016, Gill et al., 2004). 
Essas dimensões em conjunto formariam três estilos parentais: autoritário, com alta exigência e baixa responsividade; permissivo, com baixa exigência e alta responsividade; autoritativo, com alta exigência e alta responsividade (Konold \& Corneel, 2015; Gill et al., 2004). Em 1983 Maccoby e Maring acrescentaram mais um estilo, o negligente, composto por um nível baixo em ambas as dimensões. A adoção do estilo autoritativo também no contexto familiar é vista como adequada para o desenvolvimento e educação saudável dos filhos. Este estilo representa o envolvimento na educação a partir da resposta às necessidades das crianças, incentivo e diálogo, monitoramento e cobrança do cumprimento das regras, ou seja, apresenta a responsividade e da demanda (Weber, Prado, Viezzer \& Brandenburg, 2004)

Dessa forma, a presente teoria fornece como elementos chaves do clima escolar duas dimensões: estrutura disciplinar e suporte (Cornell \& Huang, 2016; Gregory et Cornell, 2009; Gregory, Cornell, Fan, Sheras, Shih, \& Huang, 2010). Tais dimensões são equiparáveis ao conceito de exigência e suporte da teoria de estilos parentais, e representam respectivamente o quanto às regras da escola são percebidas como precisas, justas e aplicadas igualmente a todos (Gerlinger \& Wo, 2016), e o quanto os estudantes percebem que seus professores e outros membros da escola os tratam com respeito e querem seu sucesso, além da identificação do estudante com a escola. (Cornell \& Huang, 2016; Huang et al., 2015; Konold \& Cornel, 2015). Assim fornecendo o modelo para ajudar a mensurar um clima positivo na escola e auxilia a clarear o entendimento dos efeitos do clima escolar (Gill et al., 2004).

É essencial que as escolas possibilitem um ambiente no qual os estudantes possam prosperar não apenas em relação aos objetivos acadêmicos, mas também como um todo. Para atingir tal fim é necessário que as escolas propiciem um ambiente no qual os estudantes sintam-se seguros (Murray-Harvey \& Slee, 2010), pois elas são ambientes fundamentais para o desenvolvimento dos estudantes de maneira integral (Opdenakker \& Minnaert, 2011). Escolas com ambientes que ofereçam estrutura disciplinar propiciam um ambiente de aprendizagem mais seguro, com menos vitimização e bullying. Oferecer, entretanto, apenas suporte ou apenas estrutura disciplinar não é suficiente, mas sim ambos para dessa maneira prover o clima autoritativo que tem um impacto mais amplo na segurança do ambiente de aprendizagem (Gregory et al., 2010).

Um ambiente com estrutura disciplinar e suporte é ideal para a promoção do desenvolvimento saudável dos estudantes, pois estes precisam de clareza das regras e 
monitoramento, mas também de adultos que compreendam sua perspectiva. Dessa forma, quando há no ambiente a estrutura disciplinar e o suporte possibilita-se o desenvolvimento da autonomia dos estudantes e a percepção deles de que a autoridade é usada de maneira adequada e não arbitrária. Além disso, esse ambiente favorece o cumprimento das regras, pois há maior possibilidade dos estudantes colaborarem e também buscarem auxílio em situações que o demandem (Gregory et al., 2010).

Um clima escolar autoritativo, portanto, é um fator protetivo que pode facilitar o desenvolvimento saudável dos estudantes (Cornell et Huang, 2016). Destaca-se, em relação a essa afirmação, que no ambiente escolar é necessário avaliar aspectos do clima não apenas relacionados ao desempenho acadêmico, mas também ao desenvolvimento sócio-emocional dos estudantes, e amplos estudos já associaram o clima escolar autoritativo a essa questão. Sendo assim, a promoção deste favorece também a aprendizagem social e emocional além da acadêmica (Bear et al., 2016).

Apesar da teoria do clima escolar autoritativo demonstrar evidências que apontam a estrutura disciplinar e o suporte ao estudante como dimensões chave para compreender o clima escolar, essa teoria não postula que essas duas dimensões abranjam todos os aspectos do clima escolar, mas sim que são características importantes que merecem um destaque na pesquisa desse contexto (Huang et Cornell, 2016; Cornell, Shukla \& Konold, 2016). A própria escala utilizada para avaliar o clima escolar autoritativo deriva da pesquisa "School Climate Bullying Survey (SCBS), a qual utiliza itens que abrangem além do suporte e estrutura disciplinar as expectativas acadêmicas, o engajamento dos estudantes, a vitimização entre pares e os valores positivos. Para o presente estudo, todavia, optou-se pela utilização da versão dessa escala que utiliza apenas os itens de estrutura disciplinar e suporte de Cornell, D., \& Huang, F. (2016), considerando que a utilização do constructo do clima escolar autoritativo a partir desses dois itens fornece um modelo promissor para investigar qualidades associadas ao clima escolar (Gregoy et al., 2010).

É importante considerar ainda, que as diferenças presentes entre os níveis de ensino podem influenciar o clima escolar (Huang \& Cornell, 2015), que também é influenciado por diferenças ambientais como as amizades, as normas e valores (Konold $\&$ Cornell, 2015). Além disso, existem indicações na literatura de que a importância dada ao clima escolar varia de escola para escola (Benbenishty,et al., 2016). 
Nesse sentido, retoma-se que o comportamento dos estudantes no ambiente escolar é influenciado por diversos fatores sociais, escolares e não escolares, mas a indisciplina, por exemplo, demonstra-se mais influenciada por fatores intra escolares. Os autores apontam a partir disso que por tal razão é necessário repensar a ideia de certa forma cristalizada no ambiente educacional de que a origem social dos estudantes se associa impreterivelmente com a indisciplina. Nesse contexto, é necessário atentar que o Brasil apresenta um sistema educacional com muita desigualdade entre as escolas. Assim é preciso que as políticas de qualidade sejam acompanhadas por políticas de equidade entre as escolas (Franco et al., 2007).

Como existem diversos elementos que podem influenciar o contexto escolar, é notório que não se pode minimizar a importância dos aspectos culturais, políticos e econômicos da educação (Del Prette e Paiva, 2005). Para o avanço da compreensão, todavia, é necessário estabelecer recortes que possibilitem a produção de conhecimento científico, como a utilização da estrutura disciplinar e do suporte como conceitos chaves para mensuração do clima escolar. Como ainda se sabe pouco sobre os preditores de um clima escolar positivo, vale o investimento para instrumentos de avaliação do construto de clima escolar autoritativo.

A maioria dos estudos sobre clima escolar, entretanto, foi conduzido em contexto estrangeiro, tendo poucas evidências sobre esse tema em outras culturas e apenas um estudo em contexto nacional. Também existem poucos instrumentos que avaliem esse construto no Brasil (Bear et al., 2016; Vinha et al., 2016). Tradicionalmente os estudos estrangeiros foram aplicados ao contexto nacional, muitos sem considerar as particularidades do Brasil como, por exemplo, as diferenças sociais, as oportunidades educacionais distintas e as diferenças entre as escolas (Berger, Lisboa, Cuadros \& Tezanos-Pinto, 2016). Ou seja, algumas pesquisas utilizam instrumentos de outras culturas, sem demonstrar a adaptação ou evidências de validade e confiabilidade para uso no contexto nacional. Dessa forma, e considerando os fatores já expostos referentes ao contexto educacional nacional faz-se necessária a existência de instrumentos que possam mensurar o clima escolar no contexto Brasileiro e assim possibilitar uma avaliação mais especifica que possa apontar intervenções em vista da melhora e potencialização do desenvolvimento saudável dos estudantes brasileiros, pois apesar de amplamente discutido em outros países, ainda não é divulgado o conhecido no contexto 
nacional. Reitera-se dessa maneira a importância do seu estudo e divulgação (Bear et al., 2016; Vinha et al., 2016).

Partindo destes pressupostos, e considerando que ainda há poucos instrumentos disponíveis em português brasileiro para a avaliação do clima escolar (Bear et al, 2016), constata-se a relevância de produzir instrumentos precisos para avaliação desse ambiente, especialmente no Brasil. Assim, o presente estudo teve como objetivos (1) realizar a adaptação para o contexto brasileiro da Escala de Avaliação do Clima Escolar Autoritativo, versão para estudantes (EACEA) (Cornell \& Huang, 2016) e (2) apresentar evidências de validade e confiabilidade deste instrumento. Nas próximas seções serão apresentados o método e resultados obtidos no processo de adaptação da EACEA para a versão brasileira.

\section{Método}

\section{Participantes}

A amostra foi constituída por 708 estudantes com idades entre 9 e 21 anos (média $=13,4 ;$ d.p. $=1,9$ ) do ensino fundamental e médio de sete escolas públicas da cidade de Curitiba, Paraná. Os participantes do ensino fundamental eram do segundo ciclo, sendo dos anos: $6^{\circ}(30,1 \%), 7^{\circ}(19,4 \%), 8^{\circ}(17,4 \%)$ e $9^{\circ}(16,8 \%)$. Do ensino médio os participantes eram do $1^{\circ}(7,6 \%), 2^{\circ}(3,7 \%)$ e $3^{\circ}(5,0 \%)$ anos. A maior parte dos estudantes frequentava o período escolar matutino $(54,5 \%)$, mas também houve participantes dos períodos vespertino $(29,4 \%)$, noturno $(9,0 \%)$ e integral $(6,8 \%)$.

A autodeclaração étnico-racial foi $55,1 \%$ brancos, $38,8 \%$ pretos e pardos $(30,3 \%$ pardos e $8,5 \%$ pretos), 3,8\% amarelos e $2,1 \%$ indígenas. Dentre os participantes, $47,0 \%$ se declararam do sexo masculino (codificado como " 0 ") e 53,0\% do sexo feminino (codificado como "1").

\section{Instrumentos}

\section{A Escala de Avaliação do Clima Escolar Autoritativo}

A Escala de Avaliação do Clima Escolar Autoritativo (EACEA), versão para estudantes (Cornell \& Huang, 2016) é um instrumento de autorrelato com objetivo de compreender como os estudantes percebem o clima escolar nessa perspectiva; que inclui dimensões como a estrutura disciplinar, suporte, mas também outros fatores, como por exemplo, seu engajamento escolar e suas expectativas acadêmicas. 
No processo de adaptação inicial foram utilizados os conceitos de suporte estrutura disciplinar, construtos que apresentam destaque nessa perspectiva e que também são utilizados em conjunto em pesquisas para avaliar comportamentos de risco e violência no ambiente escolar, como relatos de vitimização nas escolas (Cornell \& Huang, 2016).

O instrumento é composto por 15 itens e é dividido entre as dimensões de estrutura disciplinar ( 7 itens) e suporte (8 itens). Os itens sobre estrutura disciplinar refletem o quanto o estudante percebe a escola como um ambiente justo, igualitário e aberto, mas também o quanto percebe a autoridade de profissionais da escola como adequada e não coercitiva ou arbitrária (ex. "As regras da escola são justas" e "Quando os estudantes são acusados de fazer algo errado, eles têm a chance de se explicar").

Por sua, vez, o itens relativos ao suporte expressam o quanto o estudante percebe o apoio que a escola como um todo fornece, o quanto se importa com ele e tem disposição para o ajudar em casos de necessidade (ex. "A maioria dos professores e dos outros adultos da escola tratam os estudantes com respeito." e "Na escola existe um adulto com quem posso contar se eu tiver algum problema pessoal.”).

Quanto a sua forma de resposta, cada um dos itens deve ser respondido através de uma escala que indica o quanto o participante concorda com a afirmação, variando entre: $1=$ discordo muito, $2=$ discordo, $3=$ concordo, $4=$ concordo muito. A tabela 1 contém os itens e suas respectivas médias e desvio-padrão.

Tabela 1

Itens, médias e desvio-padrão dos itens da EACEA

\begin{tabular}{|c|c|c|c|c|c|}
\hline Item & Descrição & Média & d.p & Mínimo & Máximo \\
\hline$E D 1$ & $\begin{array}{l}\text { A punição por quebrar as regras da escola é a mesma } \\
\text { para todos os estudantes. }\end{array}$ & 2,89 & 0,82 & 1 & 4 \\
\hline ED2 & $\begin{array}{l}\text { Os estudantes dessa escola só são castigados quando } \\
\text { merecem. }\end{array}$ & 2,90 & 0,78 & 1 & 4 \\
\hline ED3 & $\begin{array}{l}\text { Os estudantes são tratados de forma justa, independente da } \\
\text { cor de sua pele, raça ou etnia. }\end{array}$ & 3,01 & 0,84 & 1 & 4 \\
\hline $\begin{array}{l}\text { ED4 } \\
\text { escore reverso }\end{array}$ & $\begin{array}{l}\text { Os estudantes são expulsos de sala de aula ou suspensos por } \\
\text { motivos. sem importância }\end{array}$ & 2,84 & 0,89 & 1 & 4 \\
\hline $\begin{array}{l}\text { ED5 } \\
\text { escore reverso }\end{array}$ & Os adultos dessa escola são muito rigorosos. & 2,45 & 0,82 & 1 & 4 \\
\hline ED6 & As regras da escola são justas. & 2,86 & 0,81 & 1 & 4 \\
\hline ED7 & $\begin{array}{l}\text { Quando os estudantes são acusados de fazer algo errado, } \\
\text { eles têm a chance de se explicar. }\end{array}$ & 2,85 & 0,82 & 1 & 4 \\
\hline
\end{tabular}




\begin{tabular}{|c|c|c|c|c|c|}
\hline Item & Descrição & Média & $d . p$ & Mínimo & Máximo \\
\hline$S P 1$ & $\begin{array}{l}\text { A maioria dos professores e dos outros adultos da escola se } \\
\text { importa com todos os estudantes. }\end{array}$ & 2,89 & 0,83 & 1 & 4 \\
\hline$S P 2$ & $\begin{array}{l}\text { A maioria dos professores e dos outros adultos da escola } \\
\text { querem que todos os estudantes se saiam bem nas } \\
\text { atividades. }\end{array}$ & 3,21 & 0,71 & 1 & 4 \\
\hline$S P 3$ & $\begin{array}{l}\text { A maioria dos professores e dos outros adultos da escola } \\
\text { ouvem o que os estudantes têm a dizer. }\end{array}$ & 2,74 & 0,80 & 1 & 4 \\
\hline SP4 & $\begin{array}{l}\text { A maioria dos professores e dos outros adultos da escola } \\
\text { tratam os estudantes com respeito. }\end{array}$ & 3,00 & 0,78 & 1 & 4 \\
\hline SP5 & $\begin{array}{l}\text { Na escola existe um adulto com quem posso contar se eu } \\
\text { tiver algum problema pessoal. }\end{array}$ & 2,67 & 0,99 & 1 & 4 \\
\hline SP6 & $\begin{array}{l}\text { Se eu contar ao professor que alguém está me agredindo, o } \\
\text { professor fará algo para ajudar. }\end{array}$ & 3,12 & 0,77 & 1 & 4 \\
\hline$S P 7$ & $\begin{array}{l}\text { Eu me sinto confortável em pedir ajuda a meus professores } \\
\text { para fazer as tarefas da escola. }\end{array}$ & 2,88 & 0,81 & 1 & 4 \\
\hline SP8 & $\begin{array}{l}\text { Existe pelo menos um professor ou outro adulto na escola } \\
\text { que realmente deseja que eu me vá bem nas atividades. }\end{array}$ & 3,23 & 0,71 & 1 & 4 \\
\hline
\end{tabular}

Vitimização entre pares

Por conta efeito do clima escolar sobre vitimização entre pares descrito na literatura, especialmente os conceitos de suporte e estrutura disciplinar (Cornell, Shukla, Konold, 2015; Klein, Cornell \& Konold, 2012; Vinha et al., 2016), essa dimensão foi utilizada como evidência de validade relacionado ao critério.

Foram utilizados os itens sobre vitimização da Escala de Agressão e Vitimização - EVAP (Cunha, Weber \& Steiner, 2009), um instrumento de autorrelato que investiga a agressão entre pares nos ambientes escolares. Essa dimensão é composta por oito itens nos quais o participante relata a frequência que sofreu agressão física, verbal e/ou relacional (como a exclusão social) nos últimos seis meses. As respostas são fornecidas através de uma escala Likert de cinco pontos, 1=nunca; $2=$ quase nunca; $3=$ às vezes; 4=quase sempre; $5=$ sempre. A tabela 2 apresenta os itens da escala, bem como médias e desvio-padrão dessa dimensão. 
Tabela 2

Itens, médias e desvio-padrão dos itens de vitimização da EVAP

\begin{tabular}{|c|c|c|c|c|c|}
\hline Item & Descrição & Média & d.p & Mínimo & Máximo \\
\hline VIT1 & Os colegas me provocaram & 2,44 & 1,01 & 1 & 4 \\
\hline VIT2 & $\begin{array}{l}\text { Eu fui empurrado, socado e/ou chutado por } \\
\text { colegas }\end{array}$ & 1,56 & 0,85 & 1 & 4 \\
\hline VIT3 & $\begin{array}{l}\text { Colegas ameaçaram me ferir, bater ou } \\
\text { fizeram outros tipos de ameaça }\end{array}$ & 1,45 & 0,81 & 1 & 4 \\
\hline VIT4 & $\begin{array}{l}\text { Colegas roubaram, mexeram ou estragaram } \\
\text { minhas coisas }\end{array}$ & 1,81 & 0,98 & 1 & 4 \\
\hline VIT5 & Eu fui xingado por colegas & 2,10 & 1,02 & 1 & 4 \\
\hline VIT6 & $\begin{array}{l}\text { Colegas me excluíram de grupos e /ou } \\
\text { brincadeiras }\end{array}$ & 1,63 & 0,88 & 1 & 4 \\
\hline VIT7 & $\begin{array}{l}\text { Colegas colocaram apelidos em mim que não } \\
\text { gostei }\end{array}$ & 1,92 & 1,04 & 1 & 4 \\
\hline VIT8 & $\begin{array}{l}\text { Colegas disseram coisas sobre mim para } \\
\text { fazer os outros rirem. }\end{array}$ & 1,93 & 1,00 & 1 & 4 \\
\hline
\end{tabular}

\section{Procedimentos}

Para a adaptação e validação brasileira da Escala de Avaliação do Clima Escolar Autoritativo (EACEA) (Cornell \& Huang, 2016) foram seguidos os procedimentos indicados para a validação e adaptação transcultural na literatura sobre Psicometria (Herdman, Fox-Rushby \& Badia, 1998; Hutz, Bandeira \& Trentini, 2015).

Inicialmente foi realizada a tradução dos itens do instrumento por dois juízes com conhecimento na área do estudo e proficiência em língua inglesa. Cada um deles fez uma versão da tradução do instrumento, buscando correspondência (conceitual, semântica operacional, de medida e funcional) entre os itens. As duas traduções foram comparadas e foram selecionados os itens das traduções que apresentaram maior equivalência para a composição da versão preliminar do instrumento. Seguida dessa etapa houve a retrotradução, para a comparação com o instrumento original. Esse produto foi analisado pelo grupo de pesquisa ao qual os autores fazem parte e após verificação de todos os aspectos de equivalência, o instrumento foi considerado satisfatório para aplicação.

Com a permissão das Secretarias de Educação, sete escolas foram convidadas para participação no estudo. A amostra foi selecionada por conveniência e após obtenção de autorização dos(as) gestores(as) das respectivas escolas, foi obtido consentimento 
parental ativo, bem como assentimento dos participantes antes da aplicação do questionário de forma anônima. O projeto de pesquisa foi autorizado pelo Comitê de Ética da Universidade Federal do Paraná (CAAE 54404616.0.0000.0102).

\section{Coleta de dados}

Para a coleta de dados o questionário foi disponibilizado para os participantes através de dispositivos eletrônicos (tablets). O questionário de pesquisa foi programado na ferramenta KoBoToolbox, um aplicativo desenvolvido pela Iniciativa Humanitária da Universidade de Harvard para coleta de dados (Harvard Humanitarian Initiative, n.d.). Esse software visa auxiliar a coleta de dados de maneira rápida e eficaz, sendo de uso gratuito e mantido por doações. Nas escolas a pesquisa foi respondida em grupos, sendo disponibilizado um tablet para cada participante. A instrução sobre o manuseio do dispositivo eletrônico e procedimento para responder o questionário foi feita de maneira coletiva, e os pesquisadores permaneceram em sala para auxiliar em eventuais dúvidas ou dificuldades que pudessem ocorrer. Para os estudantes que apresentaram dúvidas, os pesquisadores realizaram a leitura individual das questões.

Análise de dados

Após a etapa de coleta de dados foi realizada análise fatorial confirmatória (AFC) para avaliar a estrutura fatorial da versão brasileira. A AFC foi executada com objetivo de avaliar se o modelo proposto pela escala original também se ajustava à amostra do processo de adaptação e validação do instrumento, tal como indicado na literatura para esse processo (Field, 2009; Kline, 2011). Para a AFC foi utilizado o software Mplus, versão 6.0 (Muthén \& Muthén, 1998). Inicialmente foi criado um modelo de fator único (Clima Escolar Autoritativo), seguido do modelo com dois fatores (estrutura disciplinar e suporte).

Em seguida foi executada análise de invariância de medida entre grupos, com o objetivo de averiguar se a estrutura dos fatores apresentava diferenças entre eles. Segundo os indicadores da escala original, diferentes etapas da vida acadêmica apresentam valores distintos para as cargas fatoriais e alfas de Cronbach (Cornell, 2017). A análise seguiu a mesma divisão de estudos publicados sobre a validade da escala: os modelos foram criados com a divisão das amostras entre os $6^{\circ}, 7^{\circ}$ e $8^{\circ}$ anos do ensino fundamental (grupo 1, com $n=473$; ); e entre o $9^{\circ}$ ano do ensino fundamental, $1^{\circ}, 2^{\circ}$ e $3^{\circ}$ ano do ensino médio (grupo 2). O grupo 1 foi composto por 473 participantes (66,9\% da amostra total), com 
idades entre 9 e 18 anos (média=12,36; d.p.=1,20); sendo 51,6\% do sexo feminino (244 participantes) e 52,6\% autodeclarados brancos (249 participantes). O grupo 2 foi constituído por 273 participantes (33,1\% da amostra total); com estudantes entre 14 e 21 anos (média=15,50; d.p.=1,39); sendo 55,1\% do sexo feminino (129 participantes) e $60,3 \%$ autodeclarados brancos (141 participantes). Hipotetizou-se que diferentes faixas de agrupamento acadêmico também apresentariam diferenças, assim como nos indicadores da versão original.

Para avaliar a existência de diferenças entre os grupos nas respostas à escala foram seguidas as seguintes etapas (Damásio, 2013; Kline, 2011): (a) agrupamento entre as categorias definidas; (b) verificação de invariância métrica através do teste de restrição das cargas fatoriais para serem as mesmas entre os grupos; (c) restrição dos interceptos visando testar a invariância escalar; (d) avaliação da invariância residual dos itens através da restrição das variâncias dos erros para serem os mesmos em cada grupo; (e) avaliação da invariância estrutural com a restrição das covariâncias; (e) verificação da equivalência do construto entre os grupos através do quanto a variação do construto foi equivalente entre os grupos. Para finalizar a análise de invariância de medida, a $(f)$ correlação entre suporte e estrutura disciplinar também foi restrita para ser a mesma entre os grupos 1 e 2 , fornecendo evidências sobre as associações entre as variáveis latentes em cada um dos grupos.

Para a definição de adequação ou não do modelo foram utilizados os parâmetros das estatísticas de ajuste: comparative fit index (CFI) acima do valor de 0,9; root mean square error of approximation (RMSEA) igual ou abaixo de 0,06 e standardized root mean square residual (SRMR) abaixo de 0,08, assim como na literatura consultada (Kline, 2011). Também foi incluída a análise do qui-quadrado $\left(\chi^{2}\right)$ não significativo e sua alteração entre os modelos. Nessa forma de análise, o qui-quadrado não significativo indica que os dados e o modelo apresentam ajuste confiável, entretanto, por conta do tamanho da amostra $(n=708)$, que altera as interpretações para as diferenças de quiquadrado entre os modelos; esse indicador não se torna uma base confiável para a avaliação do modelo (Schermelleh-Engel, Moosbrugger \& Müller, 2003). Por essa razão foram utilizados primariamente os valores das estatísticas de ajuste. Ainda seguindo os parâmetros da escala original, a consistência interna foi verificada através do Alfa de Cronbach (Cronbach, 1951). Foram utilizados como parâmetros valores mínimos de 0,60 (George \& Mallery, 2003). 
Além das etapas acima, foi realizado controle para as variáveis de sexo e idade dentro de cada grupo escolar. Os valores obtidos na AFC foram comparados com os valores disponibilizados pelos autores da escala original (Cornell, 2017). Tendo em vista a inclusão de outros indicadores de validade, como a validade relacionada ao critério, foram criados modelos adicionais, comparando o efeito do clima escolar em relação à vitimização entre pares. A próxima sessão apresenta os dados obtidos nas análises realizadas.

\section{Resultados}

Análise Fatorial Confirmatória

A AFC começou pela criação do modelo de apenas um fator, denominado Clima Escolar Autoritativo (modelo 1), que abrangia todos os itens da EACEA . A Tabela 3 apresenta os valores do qui-quadrado e respectivos graus de liberdade (gl), CFI, RMSEA e SRMR para todos os modelos analisados.

O modelo 1 foi comparado com o modelo 2, com dois fatores: estrutura disciplinar e suporte. Ao avaliar ambos os modelos através dos parâmetros adotados (Kline, 2011), o modelo 2 foi considerado mais adequado por conta das estatísticas de ajuste (CFI, RMSEA, SRMR) e não da alteração do qui-quadrado $\left(\Delta \chi^{2}(1)=25,01, \mathrm{p}<0,05\right)$. Nesse modelo, os fatores estrutura disciplinar e suporte foram positivamente associados $(\mathrm{r}=0,89, \mathrm{p}<0,01)$.

Os modelos iniciais foram comparados com sete outros. No modelo 3 a amostra foi dividida para comparação entre os grupos 1 e 2. Como esse modelo não foi considerado estatisticamente aceitável, houve a remoção dos itens que apresentaram nessa etapa cargas fatoriais abaixo de 0,35 (DC3 E DC5). Com essa edição, o modelo obteve ajuste estatístico adequado, ainda que não tenha apresentado melhoria em todas as estatísticas em comparação ao modelo 2 e com alteração de qui-quadrado significativa $\left(\Delta \chi^{2}(10)=\right.$ $103,71, \mathrm{p}<0,05)$.

Seguindo as etapas estabelecidas, no modelo 4 as cargas dos fatores foram restritas para serem iguais em cada um dos grupos. Liberando 9 das 13 cargas fatoriais, o modelo apresentou melhoria nas estatísticas de ajuste, mas qui-quadrado significativo $\left(\Delta \chi^{2}{ }_{(9)}=\right.$ $33,44, \mathrm{p}<0,05)$. Portanto, apenas 4 das trezes cargas fatoriais restritas nessa etapa, o que 
indica que diferentes grupos respondem de maneiras distintas os itens da escala (Damásio, 2013), o que vai de encontro com as hipóteses iniciais de diferenças entre os grupos.

Em seguida (modelo 5) foram restritos os interceptos para serem os mesmos entre as amostras. Das 13 variáveis observáveis, a constrição de 10 apresentou melhoria ao modelo nas estatísticas de ajuste, mas não no qui-quadrado $\left(\Delta \chi^{2}(3)=13,37, \mathrm{p}<0,05\right)$. Esses resultados indicam que não houve diferenças entre os grupos para a maioria dos itens; indicando equivalência na maioria dos parâmetros da escala para ambos os agrupamentos e não apontando diferenças nos parâmetros do instrumento (Damásio, 2013).

No modelo 6 houve restrição das variâncias dos erros. Nessa etapa, a constrição de 8 das 13 variâncias não piorou o modelo em todos os indicadores $\left(\Delta \chi^{2}{ }_{(8)}=1,06, \mathrm{p}<\right.$ 0,05). Assim, para a maioria dos itens, pode-se afirmar que houve invariância dos erros entre os grupos; indicando estabilidade do instrumento nos resíduos entre as duas amostras.

Por sua vez, no modelo 7 a covariância entre os grupos foi restrita e isso significou melhoria em relação ao modelo anterior nas estatísticas de ajuste e alteração do quiquadrado $\left(\Delta \chi_{(1)}^{2}=0,98, \mathrm{p}<0,05\right)$. Esse dado indica que os construtos da escala podem apresentar a mesma disposição empírica para ambos os grupos.

As correlações entre suporte e estrutura disciplinar foram limitadas no modelo 8, mas sua restrição também significou prejuízo para o modelo nas estatísticas de ajuste, embora tenha apresentado qui-quadrado não significativo $\left(\Delta \chi^{2}(2)=0,23, p<0,05\right)$. Ainda assim o modelo foi considerado aceitável; indicando que as associações similares entre as variáveis latentes entre os grupos, mas não necessariamente iguais.

Após análise de invariância, no modelo 9 foi realizado controle para as variáveis de sexo e idade em cada um dos grupos, mantendo as constrições descritas acima. Esse modelo foi considerado aceitável e uma melhoria em relação ao anterior $\left(\Delta \chi^{2}(44)=53,03\right.$, $\mathrm{p}<0,05)$. Os fatores de estrutura disciplinar e suporte foram positivamente correlacionados $(\mathrm{r}=0,83, \mathrm{p}<0,01)$ e restritos à mesma variação entre os grupos nesse modelo, assim como descrito anteriormente. Foi observado que para os anos do grupo $1\left(6^{\circ}, 7^{\circ}\right.$ e $\left.8^{\circ}\right)$, o sexo foi um preditor negativo e significativo para a percepção do suporte $(\beta=-0,10, \mathrm{p}<$ $0,05)$. Ou seja, participantes desse grupo e do sexo masculino apresentaram associação com menores índices de percepção de suporte. 
No grupo $2\left(9^{\circ}\right.$ ano, $1^{\circ}, 2^{\circ}$ e $3^{\circ}$ ano do ensino médio) a idade foi um preditor positivo para os fatores de estrutura disciplinar $(\beta=0,18, \mathrm{p}<0,05)$ e suporte $(\beta=0,21, \mathrm{p}<$ $0,05)$; indicando que para participantes desses anos acadêmicos, a percepção mais alta da estrutura disciplinar e do suporte está associada com estudantes mais velhos. Não foram encontrados outros preditores significativos. Os resultados descritos acima indicam que há diferenças entre as etapas acadêmicas também nas associações de sexo e idade com o clima escolar.

Tabela 3

Sintese dos Modelos de AFC

\begin{tabular}{|c|c|c|c|c|c|c|}
\hline Modelo & $\chi^{2}$ & $g l$ & $C F I$ & RMSEA & $S R M R$ & Observações \\
\hline $\begin{array}{l}\text { 1) Um fator (Clima Escolar } \\
\text { Autoritativo) }\end{array}$ & 245,09 & 90 & 0,926 & 0,049 & 0,039 & Aceitável \\
\hline $\begin{array}{l}\text { 2) Dois fatores (Estrutura } \\
\text { Disciplinar e Suporte) }\end{array}$ & 220,08 & 89 & 0,937 & 0,046 & 0,037 & Aceitável* \\
\hline $\begin{array}{l}\text { 3) Dois fatores e agrupamento } \\
\text { entre as séries. Remoção das } \\
\text { variáveis DC3 e DC5 }\end{array}$ & 323,79 & 150 & 0,901 & 0,057 & 0,060 & Aceitável \\
\hline $\begin{array}{l}\text { 4) Restrição das cargas fatoriais } \\
\text { (4/13) }\end{array}$ & 290,35 & 141 & 0,915 & 0,055 & 0,049 & Aceitável \\
\hline $\begin{array}{l}\text { 5) Restrição dos interceptos } \\
\text { entre os grupos (10/13) }\end{array}$ & 276,98 & 138 & 0,921 & 0,053 & 0,047 & Aceitável \\
\hline $\begin{array}{l}\text { 6) Restrição das variâncias dos } \\
\text { erros }\end{array}$ & 278,04 & 146 & 0,925 & 0,051 & 0,047 & Aceitável \\
\hline $\begin{array}{l}\text { 7) Restrição das covariâncias } \\
\text { entre os fatores }\end{array}$ & 279,02 & 147 & 0,925 & 0,050 & 0,049 & Aceitável \\
\hline $\begin{array}{l}\text { 8) Restrição das correlações } \\
\text { entre os fatores }\end{array}$ & 279,25 & 149 & 0,926 & 0,050 & 0,050 & Aceitável \\
\hline $\begin{array}{l}\text { 9) Controle para sexo e idade } \\
\text { nos grupos }\end{array}$ & 332,28 & 193 & 0,921 & 0,045 & 0,047 & Aceitável \\
\hline \multicolumn{7}{|l|}{ Validade relacionada ao critério } \\
\hline $\begin{array}{l}\text { 10)Efeito na vitimização entre } \\
\text { pares (amostra completa) }\end{array}$ & 638,85 & 227 & 0,906 & 0,051 & 0,049 & Aceitável \\
\hline $\begin{array}{l}\text { 11)Efeito na vitimização entre } \\
\text { pares (divisão por grupos) }\end{array}$ & 1027,53 & 479 & 0,866 & 0,058 & 0,061 & Não-aceitável \\
\hline $\begin{array}{l}\text { 12) Efeito na vitimização entre } \\
\text { pares. Remoção das variáveis } \\
\text { VIT2,3,4,6. }\end{array}$ & 580,50 & 315 & 0,909 & 0,049 & 0,053 & Aceitável \\
\hline
\end{tabular}


Comparação dos dados brasileiros com dados da escala original

Os dados obtidos sobre a consistência interna e fatores foram comparados com a versão original (Cornell, 2017). A tabela 4 apresenta os valores de cada uma das versões, com descrição para cada uma das amostras.

$\mathrm{Na}$ amostra total (modelo 2) se observou que a consistência interna foi de 0,62 para o fator de estrutura disciplinar, considerando esse valor adequado, embora abaixo dos valores obtidos no estudo com a amostra original. Nesse fator, apenas uma das sete variáveis apresentou carga fatorial abaixo dos valores da escala original. Para o fator de suporte, o alfa de Cronbach também foi considerado adequado $(0,79)$ e cinco das oito variáveis apresentaram cargas fatoriais dentro da faixa estipulada pela amostra de comparação.

Ao comparar cada um dos indicadores entre os grupos (modelo 8), observa-se que não foi obtido modelo estatisticamente aceitável com todos os itens da estrutura disciplinar e que no grupo $1\left(\operatorname{anos} 6^{\circ}\right.$ ao $\left.8^{\circ}\right)$, o alfa de Cronbach para o fator de estrutura disciplinar foi inferior na versão brasileira e considerado inadequado $(0,56)$. Entretanto, as cargas fatoriais para esse fator foram mais altas do que as do instrumento original. No fator suporte, a consistência interna foi abaixo do valor de comparação, mas considerada aceitável $(0,77)$; e em cinco das oito cargas fatoriais o valor foi dentro do escopo da versão original.

Para o grupo $2\left(9^{\circ}\right.$ ano; $1^{\circ}, 2^{\circ}$ e $3^{\circ}$ anos do ensino médio), a consistência interna do fator de estrutura disciplinar também não foi aceitável $(0,59)$ e todas as cargas fatoriais desse modelo se apresentaram no mesmo escopo da escala original. No fator de suporte, a consistência interna foi dentro dos valores de comparação e novamente foi considerada adequada $(0,77)$. Dentre as cargas fatoriais, apenas três tiveram valores dentro da faixa estipulada no instrumento original. 
Tabela 4

Comparação com dados da AFC com dados da escala em outros contextos.

\begin{tabular}{|c|c|c|c|c|c|c|}
\hline \multicolumn{4}{|c|}{ Versão Brasileira } & \multicolumn{3}{|c|}{ Escala Original } \\
\hline Fator & $\begin{array}{l}\text { Alfa de } \\
\text { Cronbach }\end{array}$ & $\begin{array}{l}\text { Cargas } \\
\text { Fatoriais }\end{array}$ & Amostra & $\begin{array}{l}\text { Alfa de } \\
\text { Cronbach }\end{array}$ & $\begin{array}{l}\text { Cargas } \\
\text { Fatoriais }\end{array}$ & Amostra \\
\hline $\begin{array}{l}\text { Estrutura } \\
\text { Disciplinar }\end{array}$ & 0,62 & 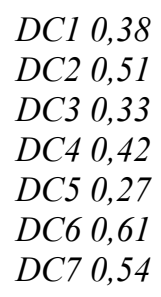 & $\begin{array}{l}\text { Total } \\
n=708 \\
\left(6^{\circ}, 7^{\circ}, 8^{\circ}, 9^{\circ} \text { anos do ensino }\right. \\
\text { fundamental e } 1^{\circ}, 2 \text { e } 3^{\circ} \text { anos } \\
\text { do ensino médio) }\end{array}$ & $0,76-0,78$ & $0,28-0,75$ & $\begin{array}{l}\text { Total } \\
n=133.789 \\
\left(6^{\circ}, 7^{\circ}, 8^{\circ}, 9^{\circ} \text { anos }\right. \\
\text { do ensino } \\
\text { fundamental e } 1^{\circ}, \\
2 \text { e } 3^{\circ} \text { anos do } \\
\text { ensino médio })\end{array}$ \\
\hline Suporte & 0,79 & $\begin{array}{ll}S P 1 & 0,69 \\
S P 2 & 0,66 \\
S P 3 & 0,66 \\
S P 4 & 0,66 \\
S P 5 & 0,47 \\
S P 6 & 0,59 \\
S P 7 & 0,47 \\
S P 8 & 0,33\end{array}$ & & $0,73-0,88$ & & $0,59-0,88$ \\
\hline $\begin{array}{l}\text { Estrutura } \\
\text { Disciplinar }\end{array}$ & 0,56 & 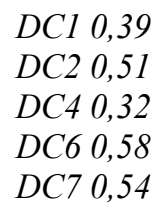 & $\begin{array}{l}6^{\circ}-8^{\circ} \text { ano } \\
n=473 \\
\text { (Grupo 1) }\end{array}$ & 0,76 & $0,28-0,74$ & $\begin{array}{l}6^{\circ}-8^{\circ} \text { ano } \\
n=85.762\end{array}$ \\
\hline Suporte & 0,77 & $\begin{array}{ll}S P 1 & 0,68 \\
S P 2 & 0,63 \\
S P 3 & 0,59 \\
S P 4 & 0,69 \\
S P 5 & 0,43 \\
S P 6 & 0,61 \\
S P 7 & 0,45 \\
S P 8 & 0,35\end{array}$ & & 0,83 & $0,59-0,88$ & \\
\hline $\begin{array}{l}\text { Estrutura } \\
\text { Disciplinar }\end{array}$ & 0,59 & 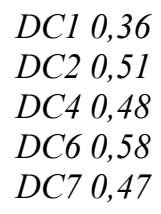 & $\begin{array}{l}9^{\circ} \text { ano e Ensino Médio } \\
n=235 \\
\text { (Grupo 2) }\end{array}$ & 0,78 & $0,36-0,75$ & $\begin{array}{l}9^{\circ} \text { ano e Ensino } \\
\text { Médio } \\
n=48.027\end{array}$ \\
\hline Suporte & 0,77 & 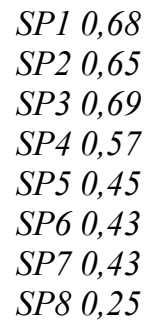 & & $0,73-0,87 * *$ & $0,63-0,87$ & \\
\hline
\end{tabular}

Nota: **Dados divididos em 2 subescalas com essa amostra 
Efeito do clima escolar em relação à vitimização entre pares

Modelos comparando o efeito dos fatores de suporte e estrutura disciplinar sobre a vitimização entre pares foram incluídos visando validade relacionada ao critério. Essa análise foi feita com a hipótese de que a estrutura disciplinar e o suporte possuiriam associações negativas com a vitimização. Foram criados modelos comparando o efeito na amostra total e na divisão dos grupos.

O modelo 10 foi criado a partir da incorporação dos itens de vitimização ao modelo 2, visando o efeito na amostra total. A variável latente vitimização foi composta por 8 itens. O suporte e a estrutura disciplinar foram colocados como preditores. Como é possível observar na tabela 3, o modelo apresentou estatísticas de ajuste aceitáveis. Foi observado efeito negativo e significativo da estrutura disciplinar na vitimização $(\beta=-0,44$, $\mathrm{p}<0,05)$, assim como hipotetizado. Todavia, não houve efeito do suporte na vitimização. Salienta-se que ao inserir as variáveis de controle de sexo e idade para a amostra completa, o modelo não foi considerado aceitável, nem com a remoção de cargas fatoriais baixas $\left(\chi^{2}{ }_{(267)}=751,67, \mathrm{p}>0,05, \mathrm{CFI}=0,891, \mathrm{RMSEA}=0,051, \mathrm{SRMR}=0,051\right)$.

Para comparar o efeito da vitimização nos grupos 1 e 2 foi criado o modelo 11 . Esse modelo teve como base o modelo 8 (com as constrições após a análise da invariância de medida) e foram adicionadas as mesmas variáveis de vitimização ao modelo. Todavia, o modelo não apresentou estatísticas de ajuste aceitáveis (ver tabela 3). Assim, para a criação do modelo 12 houve a remoção dos itens de vitimização que apresentaram as cargas fatoriais mais baixas: itens VIT2, VIT3, VIT4 E VIT6. Com essa edição o modelo apresentou estatísticas de ajuste adequado.

Não houve efeito significativo desses fatores na vitimização em relação ao grupo 1. No grupo 2 foi observado efeito negativo e significativo da estrutura disciplinar na vitimização $(\beta=-0,50, p<0,05)$; o que indica que para os estudantes das séries mais avançadas, a percepção de estrutura disciplinar foi negativamente associada com o relato da vitimização. Destaca-se que, apesar da consistência interna do fator estrutura disciplinar não ter sido adequada, a análise foi realizada utilizando as variáveis observáveis, o que permite a criação do fator, teoricamente, livre de erros (Kline, 2011). Não foram observados outros efeitos na vitimização entre pares. 
Ao comparar esses dados com dados da escala original (Cornell, 2017), também foi descrito o efeito negativo entre estrutura disciplinar e vitimização $(\beta=-0,41, p<0,05)$, mas em relação aos $7^{\circ}$ e $8^{\circ}$ anos.

\section{Discussão}

O estudo teve como objetivos realizar a adaptação dos fatores de estrutura disciplinar e suporte para a versão brasileira da EACEA (versão para estudantes) e; apresentar evidências de confiabilidade e validade do instrumento.

Os resultados da AFC demonstraram que ambos os modelos com um e dois fatores eram aceitáveis, mas que o segundo, similar à escala original, configurava melhor ajuste. $\mathrm{Na}$ amostra total, os valores de consistência interna para suporte $(0,79)$ e estrutura disciplinar $(0,62)$ foram considerados aceitáveis. Quanto às cargas fatoriais, a maioria das variáveis dessa amostra foi considerada adequada quando comparada com os dados da escala original (Cornell, 2017).

Ao realizar a divisão entre os grupos como na amostra de comparação, foram encontrados alfas de Cronbach de 0,56 (grupo 1) e 0,59 (grupo 2) para a estrutura disciplinar, considerados inadequados. Os valores de consistência interna para o suporte foram considerados adequados; 0,77 em ambas as amostras. A maior parte das cargas fatoriais das duas amostras se encontrou no mesmo escopo do instrumento original.

Após a análise de invariância de medida foi concluído que há similaridades importantes em relação a estrutura da escala nos dois grupos, mas diferenças entre eles em aspectos como, por exemplo, cargas fatoriais e resposta aos itens. Isso indica que aspectos fundamentais do instrumento, como por exemplo, a compreensão dos itens, podem estar sendo medidos da mesma forma entre os grupos, mas que eles apresentam características distintas entre si quanto aos resultados (Damásio, 2013; Kline, 2011).

Com relação a validade relacionada ao critério, observou-se que a estrutura disciplinar foi um preditor negativo e significativo da vitimização, tanto na amostra total, quanto no grupo 2. Esses dados corroboram parcialmente com estudos anteriores, (Cornell, Shukla, Konold, 2015; Klein, Cornell \& Konold, 2012), no qual o suporte e a estrutura disciplinar eram negativamente associados com a vitimização entre pares. No presente estudo se conclui que em escolas percebidas como mais justas e igualitárias pelos estudantes (Gerlinger \& Wo, 2016) houve menor associação com a vitimização entre 
pares, podendo ser esse um fator de proteção aos estudantes ao oferecer condições adequadas para interação e favorecer a colaboração com as regras da escola, em consequência auxiliar para reduzir as situações de vitimização (Vinha et al., 2016). Além disso, os resultados da comparação entre grupos sugerem que o efeito da estrutura disciplinar na vitimização parece ter mais importância conforme o estudante avança no anos da vida escolar.

Apesar das diferenças e limitações encontradas na versão brasileira, os dados obtidos indicam evidências iniciais de confiabilidade e validade da escala adaptada. Além disto, é necessário também levar em conta o tamanho e as características das amostras de comparação. A amostra brasileira, apesar de contar com 708 estudantes, não foi igual entre os grupos; com mais de $66,9 \%$ dos estudantes concentrados no grupo 1 . Não obstante, a amostra nacional possuía número de participantes bastante inferior à amostra da escala original, que contou com mais de 100 mil participantes.

Assim, além de diferenças entre os contextos, as distinções encontradas também podem ter relação com o tamanho das amostras; que por serem muito diferentes podem ter influenciado nos resultados (Field, 2009; MacCallum, Widaman, Zhang \& Hong, 1999). Visando ainda a melhoria dos indicadores avaliados, sugere-se futuros estudos com ampliação da amostra brasileira, com equiparação numérica entre os grupos. Todavia, devido a existência de poucos instrumentos que avaliem esse constructo no Brasil (Bear et al., 2016; Vinha et al., 2016) e mesmo a aplicação de instrumentos utilizados em outros países sem considerar as particularidades nacionais (Berger, Lisboa, Cuadros et Tezanos-Pinto, 2016) esse é um trabalho necessário que acrescenta para a literatura e pesquisa nacional.

Ressalta-se, contudo, que os modelos estatísticos obtidos foram aceitáveis, indicando evidências de validade e confiabilidade da versão brasileira da EACEA. Refletindo sobre a influência do clima escolar escolar no interesse na aprendizagem pelos estudantes (Carman, 2015), segurança (Murray-Harvey et Slee, 2010), engajamento escolar (Bradshaw, Waasdorp, Debnam \& Johnson,2014) e vários outros aspectos do desenvolvimento de crianças e adolescentes (Wang \& Degol, 2016), além do constructo do clima escolar autoritativo como um modelo promissor para investigar as qualidades associadas ao clima escolar (Gregoy et al., 2010), a presente escala pode ser um instrumento importante para a compreensão e para a promoção de boas práticas no ambiente escolar brasileiro 


\section{Referências}

Alves, M. T. G., \& Franco, C. (2008). A pesquisa em eficácia escolar no Brasil: evidências sobre o efeito das escolas e fatores associados à eficácia escolar. Pesquisa em eficácia escolar: origem e trajetórias. Belo Horizonte: Editora UFMG, 482-500.

Aparecida Pereira del Prette, Z., Lopez Martini Fernandes Paiva, M., \& Del Prette, A. (2005). Contribuições do referencial das habilidades sociais para uma abordagem sistêmica na compreensão do processo de ensino-aprendizagem. Interações, 10(20).

Bear, G. G., Holst, B., Lisboa, C., Chen, D., Yang, C., \& Chen, F. F. (2016). A Brazilian Portuguese survey of school climate: Evidence of validity and reliability. International Journal of School \& Educational Psychology, 4(3), 165-178.

Berger, C., Lisboa, C., Cuadros, O., \& de Tezanos-Pinto, P. (2016). Adolescent peer relations and socioemotional development in Latin America: Translating international theory into local research. New directions for child and adolescent development, 2016(152), 45-58.

Baumrind, D. (1966). Effects of authoritative control on child behavior. Child Development,37, 887-907.

Benbenishty, R., Astor, R. A., Roziner, I., \& Wrabel, S. L. (2016). Testing the Causal Links Between School Climate, School Violence, and School Academic Performance A Cross-Lagged Panel Autoregressive Model. Educational Researcher, 45(3), $197-$ 206.

Bradshaw, C. P., Waasdorp, T. E., Debnam, K. J., \& Johnson, S. L. (2014). Measuring school climate in high schools: A focus on safety, engagement, and the environment. Journalof school health, 84(9),593-604.

Carman, J. (2015). Exploring the Predictors and Outcomes of Middle School Students' Interest in Climate Change Effects on Forests: Results from Two Interventions (Doctoral dissertation).

Cohen J, McCabe E, Michelli N, Pickeral T. School climate: research, policy, practice, and teacher education. Teach Coll Rec. 2009;111(1):180-213.

Cornell, D. (2017). The authoritative school climate survey and the school climate bullying survey: Research summary. Disponível em https://curry.virginia.edu/uploads/resourceLibrary/Authoritative_School_Climate_Su rvey_Research_Summary_7-11-17.pdf

Cornell, D., \& Bradshaw, C. P. (2015). From a culture of bullying to a climate of support: The evolution of bullying prevention and research. School Psychology Review, 44(4), 499-503.

Cornell, D., \& Huang, F. (2016). Authoritative school climate and high school student risk behavior: a cross-sectional multi-level analysis of student self-reports. Journal of youth and adolescence, 45(11), 2246-2259. 
Cornell, D., Shukla, K., \& Konold, T. R. (2016). Authoritative school climate and student academic engagement, grades, and aspirations in middle and high schools. AERA Open, 2(2), 2332858416633184.

Cornell, D., Shukla, K., \& Konold, T. (2015). Peer victimization and authoritative school climate: A multilevel approach. Journal of Educational Psychology, 107(4), 1186.

Cronbach, L. J. (1951). Coefficient alpha and the internal structure of tests. psychometrika, 16(3), 297-334.

Cunha, J.M., Weber, L.N.D., \& Steiner, P. (2009) Escala de vitimização e agressão entre pares (p. 109-121). In L.N.D. Weber \& M.A. Dessen (Orgs.), Pesquisando a família: instrumentos para coleta e análise de dados. Curitiba: Juruá.

Damásio, B. F. (2013). Contribuições da Análise Fatorial Confirmatória Multigrupo (AFCMG) na avaliação de invariância de instrumentos psicométricos. Psico-Usf, $18(2), 211-220$.

Field, A. (2009). Descobrindo a estatística usando o SPSS-2. Bookman Editora.

Franco, C., Ortigão, I., Albernaz, Â., Bonamino, A., Aguiar, G., Alves, F., \& Sátyro, N. (2007). Qualidade e eqüidade em educação: reconsiderando o significado de" fatores intra-escolares". Ensaio: avaliação e políticas públicas em educação, 15(55).

Gage, N. A., Prykanowski, D. A., \& Larson, A. (2014). School climate and bullying victimization: A latent class growth model analysis. School psychology quarterly, 29(3), 256.

George, D., \& Mallery, M. (2003). Using SPSS for Windows step by step: a simple guide and reference.

Gerlinger, J., \& Wo, J. C. (2016). Preventing School Bullying: Should Schools Prioritize an Authoritative School Discipline Approach Over Security Measures?. Journal of School Violence, 15(2), 133-157.

Gill, M. G., Ashton, P., \& Algina, J. (2004). Authoritative schools: A test of a model to resolve the school effectiveness debate. Contemporary Educational Psychology, 29(4), 389-409.

Gregory, A., \& Cornell, D. (2009). "Tolerating” adolescent needs: Moving beyond zero tolerance policies in high school. Theory into Practice, 48, 106-113.

Gregory, A., Cornell, D., \& Fan, X. (2012). Teacher safety and authoritative school climate in high schools. American Journal of Education, 118(4), 401-425.

Gregory, A., Cornell, D., Fan, X., Sheras, P., Shih, T., \& Huang, F. (2010). High school practices associated with lower student bullying and victimization. Journal of Educational Psychology, 102, 483-496.

Huang, F. L., \& Cornell, D. G. (2016). Multilevel factor structure, concurrent validity, and test-retest reliability of the high school teacher version of the Authoritative School Climate Survey. Journal of Psychoeducational Assessment, 34(6), 536-549. 
Harvard Humanitarian Initiative. (n.d.). Kobo Toolbox. Retrieved November 14, 2017, from http://www.kobotoolbox.org/.

Herdman, M., Fox-Rushby, J., \& Badia, X. (1998). A model of equivalence in the cultural adaptation of HRQoL instruments: the universalist approach. Quality of life Research, 7(4), 323-335.

Huang, F. L., Cornell, D. G., Konold, T., Meyer, J. P., Lacey, A., Nekvasil, E. K., ... \& Shukla, K. D. (2015). Multilevel factor structure and concurrent validity of the teacher version of the Authoritative School Climate Survey. Journal of school health, 85(12), 843-851.

Hutz, C. S., Bandeira, D. R., \& Trentini, C. M. (2015). Psicometria. Artmed Editora.

Klein, J., Cornell, D., \& Konold, T. (2012). Relationships between bullying, school climate, and student risk behaviors. School Psychology Quarterly, 27(3), 154.

Konold, T. R., \& Cornell, D. (2015). Measurement and structural relations of an Authoritative School Climate model: A multi-level latent variable investigation. Journal of school psychology, 53(6), 447-461.

Konold, T., Cornell, D., Huang, F., Meyer, P., Lacey, A., Nekvasil, Erin,Heilbrun, Kathan.\& Shukla, K. (2014). Multilevel multi-informant structure of the Authoritative School Climate Survey. School Psychology Quarterly, 29(3), 238.

Maccoby, E. \& Martin, J. (1983). Socialization in the context of the family: parent-child interaction. Em P. H. Mussen (Org. Série) \& E. M. Hetherington (Org. Vol.), Handbook of child psychology: Vol. 4. Socialization, personality, and social development (4 a ed.)( pp. 1-101). New York: Wiley

MacCallum, R. C., Widaman, K. F., Zhang, S., \& Hong, S. (1999). Sample size in factor analysis. Psychological methods, 4(1), 84.

Murray-Harvey, R., \& Slee, P. T. (2010). School and home relationships and their impact on school bullying. School Psychology International, 31(3), 271-295.

Muthén, L. K., \& Muthén, B. O. (1998). Mplus user's guide (Vol. 6). Los Angeles, CA: Muthén \& Muthén.

Nekvasil, E. K., \& Cornell, D. G. (2015). Student threat assessment associated with safety in middle schools. Journal of Threat Assessment and Management, 2(2), 98.

Unnever, J. \& Cornell, D. (2004). Middle school victims of bullying: who reports being bullied?. Aggressive Behavior, 30, 373-388.

Oliveira, R. P., Bauer, A., Ferreira, M. P., Minuci, E. G., Lisauskas, F., Carvalho, M. X., ... \& Caldeira, C. (2013). Análise das desigualdades intraescolares no Brasil. São Paulo, SP: Fundação Vitor Civita.

Reis, R. (2012). Experiência escolar de jovens/alunos do ensino médio: os sentidos atribuídos à escola e aos estudos. Educação e Pesquisa, 38(3). 
Schermelleh-Engel, K., Moosbrugger, H., \& Müller, H. (2003). Evaluating the fit of structural equation models: Tests of significance and descriptive goodness-of-fit measures. Methods of psychological research online, 8(2), 23-74.

Stroet, K., Opdenakker, M. C., \& Minnaert, A. (2013). Effects of need supportive teaching on early adolescents' motivation and engagement: A review of the literature. Educational Research Review, 9, 65-87.

Thapa, A., Cohen, J., Guffey, S., \& Higgins-D’Alessandro, A. (2013). A review of school climate research. Review of educational research, 83(3), 357-385.

Vinha, T. P., de Morais, A., Tognetta, L. R. P., Azzi, R. G., de Aragão, A. M. F., Marques, C. D. A. E., ... \& Oliveira, M. T. A. (2016). O clima escolar e a convivência respeitosa nas instituições educativas. Estudos em Avaliação Educacional, 27(64), 96-127.

Wang, M. T., \& Degol, J. L. (2016). School climate: A review of the construct, measurement, and impact on student outcomes. Educational Psychology Review, 315-352.

Weber, L. N. D., Prado, P. M., Viezzer, A. P., \& Brandenburg, O. J. (2004). Identificação de estilos parentais: o ponto de vista dos pais e dos filhos. Psicologia: reflexão e crítica, 17(3), 323-331. 